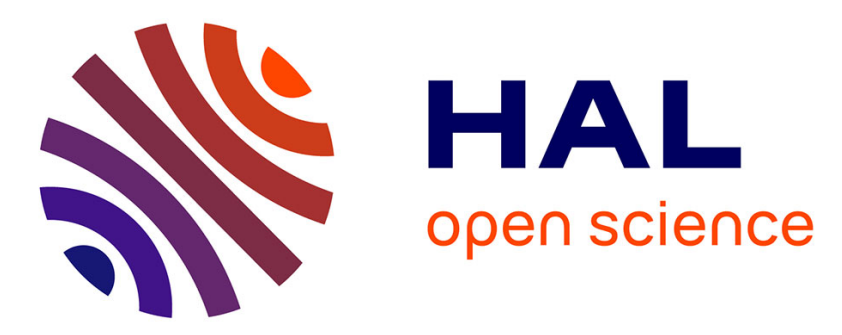

\title{
Unification with parameters in the implication fragment of Classical Propositional Logic
}

Philippe Balbiani, Mojtaba Mojtahedi

\section{To cite this version:}

Philippe Balbiani, Mojtaba Mojtahedi. Unification with parameters in the implication fragment of Classical Propositional Logic. Logic Journal of the IGPL, 2021, 10.1093/jigpal/jzab014 . hal03248073

\section{HAL Id: hal-03248073 \\ https://hal.science/hal-03248073}

Submitted on 3 Jun 2021

HAL is a multi-disciplinary open access archive for the deposit and dissemination of scientific research documents, whether they are published or not. The documents may come from teaching and research institutions in France or abroad, or from public or private research centers.
L'archive ouverte pluridisciplinaire HAL, est destinée au dépôt et à la diffusion de documents scientifiques de niveau recherche, publiés ou non, émanant des établissements d'enseignement et de recherche français ou étrangers, des laboratoires publics ou privés. 


\title{
Unification with parameters in the implication fragment of Classical Propositional Logic
}

\author{
Philippe Balbiani $^{a}$ and Mojtaba Mojtahedi ${ }^{b}$ \\ ${ }^{a}$ Toulouse Institute of Computer Science Research \\ CNRS — Toulouse University, Toulouse, France \\ ${ }^{b}$ School of Mathematics, Statistics and Computer Science \\ College of Science \\ University of Tehran, Tehran, Iran
}

\begin{abstract}
In this paper, we show that the implication fragment of Classical Propositional Logic is finitary for unification with parameters.
\end{abstract}

Keywords: Classical Propositional Logic. Implication fragment. Unification with parameters. Unification type.

\section{Introduction}

Based on a definitionally complete set of connectives, Classical Propositional Logic (denoted CPL) seems to have all good properties a propositional logic can possess. For instance, its satisfiable formulas constitutes an NP-complete set of formulas [23, Chapter 9] and its admissible rules are all derivable [9, Chapter 1]. These properties, however, are lost when the syntax is enriched as, for example, in modal logic: the satisfiable formulas defined by $\mathbf{S} 4$ constitute a PSPACE-complete set of formulas [6, Chapter 6] whereas there exists S4admissible rules that are not derivable [25, Chapter 3]. Hence, it is natural to ask what happens to the good properties of CPL when its syntax is impoverished to a definitionally incomplete set of connectives.

According to Dunn and Hardegree [11, Chapter 9], the interesting fragments of CPL are the implication fragment and the positive fragment. In this paper, we only consider the implication fragment. It is algebraically characterizable in terms of semi-Boolean algebras [1], i.e. algebraic structures of the form $(A, \triangleright)$ where $(a \triangleright b) \triangleright a=a, a \triangleright(b \triangleright c)=b \triangleright(a \triangleright c)$ and $(a \triangleright b) \triangleright b=(b \triangleright a) \triangleright a$. Its tautologies are axiomatizable by adding to the rule of modus ponens the axioms $(\varphi \rightarrow(\psi \rightarrow \chi)) \rightarrow((\varphi \rightarrow \psi) \rightarrow(\varphi \rightarrow \chi)), \varphi \rightarrow(\psi \rightarrow \varphi)$ and $((\varphi \rightarrow \psi) \rightarrow \varphi) \rightarrow \varphi$, the Pierce formula. Moreover, in it, the Deduction Theorem - if $\psi$ is derivable from $\varphi$ and a set $\Gamma$ of formulas then $\varphi \rightarrow \psi$ is derivable 
from $\Gamma$ - still holds. But what can we say about its satisfiable formulas and its admissible rules?

Concerning the satisfiable formulas, they constitute a trivial set: as the reader can prove by induction on the implicative formula $\varphi, \varphi$ is satisfiable ${ }^{1}$. Concerning the admissible rules, some of them are not derivable as soon as the language contains propositional parameters, i.e. atomic formulas that cannot be replaced by formulas when one applies substitutions. In order to understand why, consider the rule $\frac{x \rightarrow p}{p \rightarrow x}$ where $x$ is a variable and $p$ is a parameter. As the reader can prove by induction on the implicative formula $\varphi$, if $\varphi \rightarrow p$ is valid then $p \rightarrow \varphi$ is valid. Hence, the considered rule is admissible. Nevertheless, it is not derivable. Indeed, suppose the considered rule is derivable. Thus, by using the Deduction Theorem, the formula $(x \rightarrow p) \rightarrow(p \rightarrow x)$ is valid: a contradiction.

In a propositional $\operatorname{logic} \mathbf{L}$, the admissibility problem is related to the unifiability problem [16, 17]: given a formula $\varphi$, determine whether it has an $\mathbf{L}$-unifier, i.e. a substitution $\sigma$ such that $\sigma(\varphi)$ is in $\mathbf{L}$. A unifiable formula is finitary if it has a finite complete set of unifiers and unitary if this set is a singleton. It is well-known that, based on a definitionally complete set of connectives, CPL is unitary $[4,13,22]$. We usually distinguish between elementary unification when all atomic formulas are considered as variables - and unification with parameters - when some atomic formulas are considered as parameters.

It may happen that in a propositional logic, every parameter-free unifiable formula is unitary whereas some unifiable formulas with parameters are finitary. For instance, in [15], Ghilardi has shown that the implication-conjunction fragment of Intuitionistic Propositional Logic is unitary for elementary unification and finitary for unification with parameters. From [10, 24], we know that the implication fragment of every intermediate logic is unitary for elementary unification. In this paper, we show that the implication fragment of Classical Propositional Logic is finitary for unification with parameters.

\section{Classical Propositional Logic and its implica- tion fragment}

Atoms and models Let VAR be a countable set of variables (with typical members denoted $x, y$, etc) and PAR be a countable set of parameters (with typical members denoted $p, q$, etc). Atoms (denoted $\alpha, \beta$, etc) are variables or parameters. Let ATO be the set of all atoms. A model is a function of the form

- $V:$ ATO $\longrightarrow\{0,1\}$.

\footnotetext{
${ }^{1}$ Concerning the valid formulas, they constitute a coNP-hard set. Why? Simply because for all formulas $F$ in the definitionally complete set $\{\neg, \rightarrow\}$ of connectives and for all atomic formulas $\alpha$ not occurring in $F, F$ is valid if and only if the implicative formula $(\varphi \rightarrow \alpha) \rightarrow \alpha$ is valid, $\varphi$ being the implicative formula obtained from $F$ after having inductively replaced each subformula of the form $\neg H$ by $H \rightarrow \alpha$.
} 
Formulas Formulas (denoted $\varphi, \psi$, etc) are inductively defined as follows:

- $\varphi, \psi::=\alpha \mid(\varphi \rightarrow \psi)$.

Let FOR be the set of all formulas. We adopt the standard rules for omission of the parentheses. The Boolean connective of disjunction is defined, at the level of formulas, as follows:

- $(\varphi \vee \psi)::=((\varphi \rightarrow \psi) \rightarrow \psi)$.

For all formulas $\varphi$, let $\operatorname{var}(\varphi)$ be the set of all variables occurring in $\varphi$ and $\operatorname{par}(\varphi)$ be the set of all parameters occurring in $\varphi$. We shall say that a formula $\varphi$ is variable-free if $\operatorname{var}(\varphi)=\emptyset$ and parameter-free if $\operatorname{par}(\varphi)=\emptyset$. For all finite $S \subseteq$ FOR, let $\operatorname{var}(S)$ be $\bigcup\{\operatorname{var}(\varphi): \varphi \in S\}$ and $\operatorname{par}(S)$ be $\bigcup\{\operatorname{par}(\varphi): \varphi \in S\}$. Given a model $V:$ ATO $\longrightarrow\{0,1\}$, the function $\bar{V}: \mathbf{F O R} \longrightarrow\{0,1\}$ is inductively defined as follows:

- $\bar{V}(\alpha)=V(\alpha)$,

- $\bar{V}(\varphi \rightarrow \psi)=\max \{1-\bar{V}(\varphi), \bar{V}(\psi)\}$.

As a result,

- $\bar{V}(\varphi \vee \psi)=\max \{\bar{V}(\varphi), \bar{V}(\psi)\}$.

A formula $\varphi$ is valid (in symbols $\models \varphi$ ) if for all models $V, \bar{V}(\varphi)=1$. A finite set $S$ of formulas is valid (in symbols $\models S$ ) if for all $\varphi \in S, \models \varphi$.

Proposition 1 For all formulas $\varphi$, there exists an atom $\alpha$ such that $\models \alpha \rightarrow \varphi$.

Proof: By induction on $\varphi$. $\dashv$

Proposition 2 Let $\alpha$ be an atom and $\varphi$ be a formula. For all $n \geq 1$ and for all atoms $\beta_{1}, \ldots, \beta_{n}$,

1. if $\models \alpha \rightarrow \beta_{1} \vee \ldots \vee \beta_{n}$ then there exists $i \in\{1, \ldots, n\}$ such that $\alpha=\beta_{i}$,

2. if $=\varphi \rightarrow \beta_{1} \vee \ldots \vee \beta_{n}$ then there exists $i \in\{1, \ldots, n\}$ such that $\models \beta_{i} \rightarrow \varphi$.

Proof: Let $n \geq 1$ and $\beta_{1}, \ldots, \beta_{n}$ be atoms.

(1). Left to the reader.

(2). Suppose $\models \varphi \rightarrow \beta_{1} \vee \ldots \vee \beta_{n}$. By Proposition 1, let $\alpha$ be an atom such that $\models \alpha \rightarrow \varphi$. Since $\models \varphi \rightarrow \beta_{1} \vee \ldots \vee \beta_{n}, \mid=\alpha \rightarrow \beta_{1} \vee \ldots \vee \beta_{n}$. Hence, by Item (1), let $i \in\{1, \ldots, n\}$ be such that $\alpha=\beta_{i}$. Since $\models \alpha \rightarrow \varphi, \models \beta_{i} \rightarrow \varphi$. $\dashv$ 
Extended formulas Extended formulas (denoted $F, G$, etc) are inductively defined as follows:

- $F, G::=\alpha|(F \rightarrow G)| \neg F$.

Let EXT be the set of all extended formulas. We adopt the standard rules for omission of the parentheses. The Boolean connectives of disjunction, conjunction and equivalence are defined, at the level of extended formulas, as usual. For all extended formulas $F$, let $\operatorname{var}(F)$ be the set of all variables occurring in $F$ and $\operatorname{par}(F)$ be the set of all parameters occurring in $F$. We shall say that an extended formula $F$ is variable-free if $\operatorname{var}(F)=\emptyset$ and parameterfree if $\operatorname{par}(F)=\emptyset$. Given a model $V:$ ATO $\longrightarrow\{0,1\}$, the function $\bar{V}: \mathbf{E X T} \longrightarrow\{0,1\}$ is inductively defined as usual. An extended formula $F$ is valid (in symbols $=F$ ) if for all models $V, \bar{V}(F)=1$. We shall say that the extended formula $F$ is logically equivalent to the extended formula $G$ if $\models F \leftrightarrow G$.

A useful result Obviously, every formula is an extended formula. We shall say that an extended formula is implicative if it is logically equivalent to a formula. For all atoms $\alpha$, when we write $\alpha^{0}$ and $\alpha^{1}$, we respectively mean $\neg \alpha$ and $\alpha$.

Proposition 3 Let $\alpha$ be an atom. For all $k \in \mathbb{N}$, for all atoms $\beta_{1}, \ldots, \beta_{k}$ and for all $a_{1}, \ldots, a_{k} \in\{0,1\}, \bigwedge\left\{\beta_{j}^{a_{j}}: 1 \leq j \leq k\right\} \vee \alpha$ is implicative.

Proof: Let $k \in \mathbb{N}, \beta_{1}, \ldots, \beta_{k}$ be atoms and $a_{1}, \ldots, a_{k} \in\{0,1\}$. Obviously, $\models\left(\bigwedge\left\{\beta_{j}^{a_{j}}: 1 \leq j \leq k\right\} \vee \alpha\right) \leftrightarrow\left(\bigvee\left\{\beta_{j}^{1-a_{j}}: 1 \leq j \leq k\right\} \rightarrow \alpha\right)$. Hence, $\models$ $\left(\bigwedge\left\{\beta_{j}^{a_{j}}: 1 \leq j \leq k\right\} \vee \alpha\right) \leftrightarrow\left(\bigvee\left\{\beta_{j}: 1 \leq j \leq k\right.\right.$ and $\left.a_{j}=0\right\} \vee \bigvee\left\{\neg \beta_{j}: 1 \leq j \leq k\right.$ and $\left.\left.a_{j}=1\right\} \rightarrow \alpha\right)$. Thus, $\models\left(\bigwedge\left\{\beta_{j}^{a_{j}}: 1 \leq j \leq k\right\} \vee \alpha\right) \leftrightarrow\left(\bigvee\left\{\beta_{j}: 1 \leq j \leq k\right.\right.$ and $\left.a_{j}=0\right\} \vee \bigvee\left\{\beta_{j} \rightarrow \alpha: 1 \leq j \leq k\right.$ and $\left.\left.a_{j}=1\right\} \rightarrow \alpha\right)$. Consequently, $\bigwedge\left\{\beta_{j}^{a_{j}}: 1 \leq j \leq k\right\} \vee \alpha$ is implicative ${ }^{2}$. $\dashv$

Proposition 4 For all extended formulas $F$, the following conditions are equivalent:

1. F is implicative,

2. there exists an atom $\alpha$ such that $=\alpha \rightarrow F$,

3. there exists an extended formula $G$ and there exists an atom $\alpha$ such that $=F \leftrightarrow(G \vee \alpha)$,

4. there exists an extended formula $G$ and there exists an atom $\alpha$ such that $=F \leftrightarrow(G \rightarrow \alpha)$.

\footnotetext{
${ }^{2}$ In this argument, we assume $k \geq 1$. The truth is that if $k=0$ then $\bigwedge\left\{\beta_{j}^{a_{j}}: 1 \leq j \leq k\right\} \vee \alpha$ is implicative, seeing that $\models\left(\bigwedge\left\{\beta_{j}^{a_{j}}: 1 \leq j \leq k\right\} \vee \alpha\right) \leftrightarrow(\alpha \rightarrow \alpha)$.
} 
Proof: Let $F$ be an extended formula.

$(1 \Rightarrow 2)$. Suppose $F$ is implicative. Let $\varphi$ be a formula such that $=F \leftrightarrow \varphi$. By Proposition 1, let $\alpha$ be an atom such that $\models \alpha \rightarrow \varphi$. Since $\models F \leftrightarrow \varphi, \models \alpha \rightarrow F$.

$(2 \Rightarrow 3)$. Suppose there exists an atom $\alpha$ such that $\models \alpha \rightarrow F$. Hence, $\models F \leftrightarrow(F \vee \alpha)$.

$(3 \Rightarrow 4)$. Suppose there exists an extended formula $G$ and there exists an atom $\alpha$ such that $\models F \leftrightarrow(G \vee \alpha)$. Hence, $\models F \leftrightarrow(\neg G \rightarrow \alpha)$.

$(4 \Rightarrow 1)$. Suppose there exists an extended formula $G$ and there exists an atom $\alpha$ such that $=F \leftrightarrow(G \rightarrow \alpha)$. Let $H$ be a conjunctive normal form of $G$, i.e. let $n \in \mathbb{N}, k_{1}, \ldots, k_{n} \in \mathbb{N}, \beta_{1,1}, \ldots, \beta_{1, k_{1}}, \ldots, \beta_{n, 1}, \ldots, \beta_{n, k_{n}} \in \mathbf{A T O}$ and $a_{1,1}, \ldots, a_{1, k_{1}}, \ldots, a_{n, 1}, \ldots, a_{n, k_{n}} \in\{0,1\}$ be such that $H=\bigwedge\left\{\bigvee\left\{\beta_{i, j}^{a_{i, j}}\right.\right.$ : $\left.\left.1 \leq j \leq k_{i}\right\}: 1 \leq i \leq n\right\}$ and $\mid=G \leftrightarrow H$. Since $=F \leftrightarrow(G \rightarrow \alpha)$, $\models F \leftrightarrow\left(\bigwedge\left\{\bigvee\left\{\beta_{i, j}^{a_{i, j}}: 1 \leq j \leq k_{i}\right\}: 1 \leq i \leq n\right\} \rightarrow \alpha\right)$. Hence, $\models F \leftrightarrow$ $\left(\bigvee\left\{\bigwedge\left\{\beta_{i, j}^{1-a_{i, j}}: 1 \leq j \leq k_{i}\right\}: 1 \leq i \leq n\right\} \vee \alpha\right)$. Thus, $\models F \leftrightarrow \bigvee\left\{\bigwedge\left\{\beta_{i, j}^{1-a_{i, j}}:\right.\right.$ $\left.\left.1 \leq j \leq k_{i}\right\} \vee \alpha: 1 \leq i \leq n\right\}$. Consequently, by Proposition $3, F$ is implicative ${ }^{3}$. $\dashv$

\section{Unification with parameters}

Substitutions A substitution is a function of the form

- $\sigma:$ VAR $\longrightarrow$ FOR.

We shall say that a substitution $\sigma$ moves a variable $x$ if $\sigma(x) \neq x$. Following the standard assumption considered in the literature about unification $[2,5]$, we will always assume that substitutions move at most finitely many variables. Let SUB be the set of all substitutions. For all finite $X \subseteq$ VAR, an $X$ substitution is a substitution that does not move the variables in VAR $\backslash X$. For all formulas $\varphi\left(x_{1}, \ldots, x_{n}\right)$ and for all substitutions $\sigma$, let $\sigma\left(\varphi\left(x_{1}, \ldots, x_{n}\right)\right)$ be $\varphi\left(\sigma\left(x_{1}\right), \ldots, \sigma\left(x_{n}\right)\right)$. For all finite $S \subseteq \mathbf{F O R}$ and for all substitutions $\sigma$, let $\sigma(S)$ be $\{\sigma(\varphi): \varphi \in S\}$. For all extended formulas $F\left(x_{1}, \ldots, x_{n}\right)$ and for all substitutions $\sigma$, let $\sigma\left(F\left(x_{1}, \ldots, x_{n}\right)\right)$ be $F\left(\sigma\left(x_{1}\right), \ldots, \sigma\left(x_{n}\right)\right)$. The composition $\sigma \circ \tau$ of the substitutions $\sigma$ and $\tau$ is the substitution associating to each variable $x$ the formula $\tau(\sigma(x))$. The identity substitution (denoted id) is the substitution associating to each variable $x$ the formula $x$. For all $n \in \mathbb{N}$ and for all substitutions $\sigma$, the substitution $\sigma^{n}$ is inductively defined as follows:

- $\sigma^{0}=\mathrm{id}$

- $\sigma^{n+1}=\sigma^{n} \circ \sigma$.

\footnotetext{
${ }^{3}$ In this argument, we assume $n \geq 1$. The truth is that if $n=0$ then $F$ is implicative, seeing that $\models F \leftrightarrow \alpha$.
} 
Proposition 5 For all $n \in \mathbb{N}$ and for all substitutions $\sigma, \sigma \circ \sigma^{n}=\sigma^{n} \circ \sigma$.

Proof: By induction on $n$. $\dashv$

For all finite $X \subseteq \mathbf{V A R}$, the equivalence relation $\simeq_{X}$ on SUB is defined by

- $\sigma \simeq_{X} \tau$ if and only if for all $x \in X, \models \sigma(x) \leftrightarrow \tau(x)$.

For all finite $X \subseteq$ VAR, the quasi-order $\preceq_{X}$ on SUB is defined by

- $\sigma \preceq_{X} \tau$ if and only if there exists $v \in$ SUB such that for all $x \in X$, $=v(\sigma(x)) \leftrightarrow \tau(x)$.

Useful results Let $k \in \mathbb{N}$. Let $\mathcal{S}=\{0,1\}^{k}$ (with typical members denoted $\left(a_{1}, \ldots, a_{k}\right),\left(b_{1}, \ldots, b_{k}\right)$, etc) and $\ll$ be the binary relation on $\mathcal{S}$ defined as follows:

- $\left(a_{1}, \ldots, a_{k}\right) \ll\left(b_{1}, \ldots, b_{k}\right)$ iff for all $i \in\{1, \ldots, k\}, a_{i} \leq b_{i}$.

Obviously, $(\mathcal{S}, \ll)$ is a finite lattice in which the length of an arbitrary chain is at most equal to $k$.

Proposition 6 Let $\left\{y_{1}, \ldots, y_{k}\right\}$ be a finite set of variables and $\sigma$ be a substitution such that for all $i \in\{1, \ldots, k\}, \operatorname{var}\left(\sigma\left(y_{i}\right)\right) \subseteq\left\{y_{1}, \ldots, y_{k}\right\}$. Let $\varphi$ be a variable-free formula such that for all $i \in\{1, \ldots, k\}, \models \varphi \rightarrow\left(y_{i} \rightarrow \sigma\left(y_{i}\right)\right)$. For all $n \in \mathbb{N}$ and for all $j \in\{1, \ldots, k\}$, if $n \geq k$ then $\models \varphi \rightarrow\left(\sigma\left(\sigma^{n}\left(y_{j}\right)\right) \leftrightarrow \sigma^{n}\left(y_{j}\right)\right)$.

Proof: Let $n \in \mathbb{N}$ and $j \in\{1, \ldots, k\}$ be such that $n \geq k$. For the sake of the contradiction, suppose $\not \models \varphi \rightarrow\left(\sigma\left(\sigma^{n}\left(y_{j}\right)\right) \leftrightarrow \sigma^{n}\left(y_{j}\right)\right)$. Let $V$ be a model such that $\bar{V}(\varphi)=1$ and $\bar{V}\left(\sigma\left(\sigma^{n}\left(y_{j}\right)\right)\right)$ and $\bar{V}\left(\sigma^{n}\left(y_{j}\right)\right)$ are not equal. Since for all $i \in\{1, \ldots, k\}, \operatorname{var}\left(\sigma\left(y_{i}\right)\right) \subseteq\left\{y_{1}, \ldots, y_{k}\right\}$, for all models $V^{\prime}, V^{\prime \prime}$, if $V_{\mid \mathbf{P A R}}^{\prime}=$ $V_{\mid \mathbf{P A R}}^{\prime \prime}$ and for all $i \in\{1, \ldots, k\}, V^{\prime}\left(y_{i}\right)=V^{\prime \prime}\left(y_{i}\right)$ then for all $i \in\{1, \ldots, k\}$, $\bar{V}^{\prime}\left(\sigma\left(y_{i}\right)\right)=\bar{V}^{\prime \prime}\left(\sigma\left(y_{i}\right)\right)$. For all $\left(a_{1}, \ldots, a_{k}\right) \in \mathcal{S}$, let $f\left(\left(a_{1}, \ldots, a_{k}\right)\right)$ be the unique $\left(b_{1}, \ldots, b_{k}\right) \in \mathcal{S}$ such that for all models $V^{\prime}$, if $V_{\mid \mathbf{P A R}}^{\prime}=V_{\mid \mathbf{P A R}}$ and for all $i \in\{1, \ldots, k\}, V^{\prime}\left(y_{i}\right)=a_{i}$ then for all $i \in\{1, \ldots, k\}, \bar{V}^{\prime}\left(\sigma\left(y_{i}\right)\right)=b_{i}$. Since $\varphi$ is variable-free, for all $i \in\{1, \ldots, k\}, \models \varphi \rightarrow\left(y_{i} \rightarrow \sigma\left(y_{i}\right)\right)$ and $\bar{V}(\varphi)=1$, for all $\left(a_{1}, \ldots, a_{k}\right) \in \mathcal{S},\left(a_{1}, \ldots, a_{k}\right) \ll f\left(\left(a_{1}, \ldots, a_{k}\right)\right)$. Since the length of an arbitrary chain in $(\mathcal{S}, \ll)$ is at most equal to $k$ and $n \geq k, f\left(f^{n}\left(\left(V\left(y_{1}\right), \ldots, V\left(y_{k}\right)\right)\right)\right)=$ $f^{n}\left(\left(V\left(y_{1}\right), \ldots, V\left(y_{k}\right)\right)\right)$. Hence, $\quad\left(\bar{V}\left(\sigma\left(\sigma^{n}\left(y_{1}\right)\right)\right), \ldots, \bar{V}\left(\sigma\left(\sigma^{n}\left(y_{k}\right)\right)\right)\right)=$ $\left(\bar{V}\left(\sigma^{n}\left(y_{1}\right)\right), \ldots, \bar{V}\left(\sigma^{n}\left(y_{k}\right)\right)\right)$. Thus, $\bar{V}\left(\sigma\left(\sigma^{n}\left(y_{j}\right)\right)\right)$ and $\bar{V}\left(\sigma^{n}\left(y_{j}\right)\right)$ are equal: a contradiction. $\dashv$

Proposition 7 Let $\left\{y_{1}, \ldots, y_{k}\right\}$ be a finite set of variables and $\sigma$ be a substitution such that for all $i \in\{1, \ldots, k\}, \operatorname{var}\left(\sigma\left(y_{i}\right)\right) \subseteq\left\{y_{1}, \ldots, y_{k}\right\}$. Let $\varphi$ be a variable-free formula such that for all $i \in\{1, \ldots, k\}, \models \varphi \vee\left(\sigma\left(y_{i}\right) \rightarrow y_{i}\right)$. For all $n \in \mathbb{N}$ and for all $j \in\{1, \ldots, k\}$, if $n \geq k$ then $=\varphi \vee\left(\sigma\left(\sigma^{n}\left(y_{j}\right)\right) \leftrightarrow \sigma^{n}\left(y_{j}\right)\right)$. 
Proof: Let $n \in \mathbb{N}$ and $j \in\{1, \ldots, k\}$ be such that $n \geq k$. For the sake of the contradiction, suppose $\not \models \varphi \vee\left(\sigma\left(\sigma^{n}\left(y_{j}\right)\right) \leftrightarrow \sigma^{n}\left(y_{j}\right)\right)$. Let $V$ be a model such that $\bar{V}(\varphi)=0$ and $\bar{V}\left(\sigma\left(\sigma^{n}\left(y_{j}\right)\right)\right)$ and $\bar{V}\left(\sigma^{n}\left(y_{j}\right)\right)$ are not equal. Since for all $i \in\{1, \ldots, k\}, \operatorname{var}\left(\sigma\left(y_{i}\right)\right) \subseteq\left\{y_{1}, \ldots, y_{k}\right\}$, for all models $V^{\prime}, V^{\prime \prime}$, if $V_{\mid \mathbf{P A R}}^{\prime}=$ $V_{\mid \mathbf{P A R}}^{\prime \prime}$ and for all $i \in\{1, \ldots, k\}, V^{\prime}\left(y_{i}\right)=V^{\prime \prime}\left(y_{i}\right)$ then for all $i \in\{1, \ldots, k\}$, $\bar{V}^{\prime}\left(\sigma\left(y_{i}\right)\right)=\bar{V}^{\prime \prime}\left(\sigma\left(y_{i}\right)\right)$. For all $\left(a_{1}, \ldots, a_{k}\right) \in \mathcal{S}$, let $f\left(\left(a_{1}, \ldots, a_{k}\right)\right)$ be the unique $\left(b_{1}, \ldots, b_{k}\right) \in \mathcal{S}$ such that for all models $V^{\prime}$, if $V_{\mid \mathbf{P A R}}^{\prime}=V_{\mid \mathbf{P A R}}$ and for all $i \in\{1, \ldots, k\}, V^{\prime}\left(y_{i}\right)=a_{i}$ then for all $i \in\{1, \ldots, k\}, \bar{V}^{\prime}\left(\sigma\left(y_{i}\right)\right)=b_{i}$. Since $\varphi$ is variable-free, for all $i \in\{1, \ldots, k\},=\varphi \vee\left(\sigma\left(y_{i}\right) \rightarrow y_{i}\right)$ and $\bar{V}(\varphi)=0$, for all $\left(a_{1}, \ldots, a_{k}\right) \in \mathcal{S}, f\left(\left(a_{1}, \ldots, a_{k}\right)\right) \ll\left(a_{1}, \ldots, a_{k}\right)$. Since the length of an arbitrary chain in $(\mathcal{S}, \ll)$ is at most equal to $k$ and $n \geq k, f\left(f^{n}\left(\left(V\left(y_{1}\right), \ldots, V\left(y_{k}\right)\right)\right)\right)=$ $f^{n}\left(\left(V\left(y_{1}\right), \ldots, V\left(y_{k}\right)\right)\right)$. Hence, $\quad\left(\bar{V}\left(\sigma\left(\sigma^{n}\left(y_{1}\right)\right)\right), \ldots, \bar{V}\left(\sigma\left(\sigma^{n}\left(y_{k}\right)\right)\right)\right)=$ $\left(\bar{V}\left(\sigma^{n}\left(y_{1}\right)\right), \ldots, \bar{V}\left(\sigma^{n}\left(y_{k}\right)\right)\right)$. Thus, $\bar{V}\left(\sigma\left(\sigma^{n}\left(y_{j}\right)\right)\right)$ and $\bar{V}\left(\sigma^{n}\left(y_{j}\right)\right)$ are equal: a contradiction. $\dashv$

Unifiers Let $S \subseteq$ FOR be finite. A unifier of $S$ is a $\operatorname{var}(S)$-substitution $\sigma$ such that $=\sigma(S)$. $S$ is unifiable if there exists a unifier of $S$.

Proposition 8 For all unifiers $\sigma$ of $S$, there exists a unifier $\tau$ of $S$ such that $\tau \preceq_{\operatorname{var}(S)} \sigma$ and $\operatorname{par}(\tau(S)) \subseteq \operatorname{par}(S)$.

Proof: Let $\sigma$ be a unifier of $S$. Hence, $\models \sigma(S)$. Let $q_{1}, \ldots, q_{l}$ be the list of the parameters $q$ such that $q \in \operatorname{par}(\sigma(S)) \backslash \operatorname{par}(S)$. Let $z_{1}, \ldots, z_{l}$ be new variables. Let $\tau$ be the substitution obtained from $\sigma$ by respectively replacing in $\sigma(x)$ each occurrence of $q_{1}, \ldots, q_{l}$ by $z_{1}, \ldots, z_{l}$ for each variable $x$ in $S$. Obviously, $\tau \preceq_{\operatorname{var}(S)} \sigma$. Moreover, since $\models \sigma(S), \models \tau(S)$. Thus, $\tau$ is a unifier of $S$. In other respect, obviously, $\operatorname{par}(\tau(S)) \subseteq \operatorname{par}(S)$. $\dashv$

Unification types Let $S \subseteq$ FOR be finite. If $S$ is unifiable then a set $\Sigma$ of unifiers of $S$ is complete if for all unifiers $\sigma$ of $S$, there exists $\tau \in \Sigma$ such that $\tau \preceq_{\operatorname{var}(S)} \sigma$. As is well-known, if $S$ is unifiable then for all minimal complete sets $\Sigma, \Delta$ of unifiers of $S, \operatorname{Card}(\Sigma)=\operatorname{Card}(\Delta)^{4}$. As a result, if $S$ is unifiable then

- $S$ is unitary if there exists a minimal complete set of unifiers of $S$ with cardinality 1 ,

- $S$ is finitary if there exists a minimal complete set of unifiers of $S$ with finite cardinality $\geq 2$,

- $S$ is infinitary if there exists a minimal complete set of unifiers of $S$ with infinite cardinality,

\footnotetext{
${ }^{4}$ This is a standard result, although we have not been able to find a reference proving it. Suppose $S$ is unifiable. Let $\Sigma, \Delta$ be minimal complete sets of unifiers of $S$. By the completeness of $\Sigma$ and $\Delta$, one can readily define functions $f: \Sigma \longrightarrow \Delta$ and $g: \Delta \longrightarrow \Sigma$ such that $f(\sigma) \preceq_{\operatorname{var}(S)} \sigma$ for each $\sigma \in \Sigma$ and $g(\delta) \preceq_{\operatorname{var}(S)} \delta$ for each $\delta \in \Delta$. By the minimality of $\Sigma$ and $\Delta$, it easily follows that $f$ and $g$ are injective. Thus, $\Sigma$ and $\Delta$ have the same cardinality.
} 
- $S$ is nullary if there exists no minimal complete set of unifiers of $S$.

\section{About the type of the implication fragment}

Let $S \subseteq$ FOR be finite. Assume $\operatorname{par}(S) \neq \emptyset$ and $S$ is unifiable. Let $\sigma$ be a unifier of $S$. By Proposition 8, let $\tau$ be a unifier of $S$ such that $\tau \preceq \operatorname{var}(S) \sigma$ and $\operatorname{par}(\tau(S)) \subseteq \operatorname{par}(S)$. Since $\operatorname{par}(S) \neq \emptyset$, let $p_{0}$ be a parameter in $S$. Let $\mu$ be the substitution defined as follows:

- for all variables $x$, if there exists $y \in \operatorname{var}(S)$ such that $x \in \operatorname{var}(\tau(y))$ then $\mu(x)=p_{0} \rightarrow p_{0}$,

- for all variables $x$, if for all $y \in \operatorname{var}(S), x \notin \operatorname{var}(\tau(y))$ then $\mu(x)=x$.

Note that $\operatorname{var}(\mu(\tau(S)))=\emptyset$. For all $x \in \operatorname{var}(S)$, by Proposition 4, there exists an atom $\alpha_{x}$ in $\tau(x)$ such that $\models \alpha_{x} \rightarrow \tau(x)$. For all $x \in \operatorname{var}(S)$, let $F_{x}$ be the extended formula defined as follows:

- if $\alpha_{x}$ is a variable then let $F_{x}=\neg\left(p_{0} \rightarrow p_{0}\right)$,

- if $\alpha_{x}$ is a parameter then let $F_{x}=\alpha_{x}$.

Note that for all $x \in \operatorname{var}(S), F_{x}$ is variable-free.

Lemma 1 For all $x \in \operatorname{var}(S)$,

$$
\begin{aligned}
& \text { 1. }=F_{x} \rightarrow \tau(x), \\
& \text { 2. }=F_{x} \rightarrow \mu(\tau(x)) .
\end{aligned}
$$

Proof: Let $x \in \operatorname{var}(S)$.

(1). Case $\alpha_{x}$ is a variable: Hence, $F_{x}=\neg\left(p_{0} \rightarrow p_{0}\right)$. Thus, $\models F_{x} \rightarrow \tau(x)$.

Case $\alpha_{x}$ is a parameter: Hence, $F_{x}=\alpha_{x}$. Since $\models \alpha_{x} \rightarrow \tau(x), \models F_{x} \rightarrow \tau(x)$.

(2). By item $1, \models F_{x} \rightarrow \tau(x)$. Hence, $\models F_{x} \rightarrow \mu(\tau(x))$. $\dashv$

For all $x \in \operatorname{var}(S)$, let $G_{x}=(\bigwedge S \wedge x) \vee(\neg \bigwedge S \wedge \mu(\tau(x))) \vee F_{x}{ }^{5}$.

Lemma 2 For all $x \in \operatorname{var}(S), G_{x}$ is implicative.

Proof: Let $x \in \operatorname{var}(S)$.

Case $\alpha_{x}$ is a variable: Hence, $F_{x}=\neg\left(p_{0} \rightarrow p_{0}\right)$. Thus, $\models G_{x} \leftrightarrow(\bigwedge S \wedge$ $x) \vee(\neg \bigwedge S \wedge \mu(\tau(x)))$. Since $\alpha_{x}$ is an atom in $\tau(x), \mu\left(\alpha_{x}\right)=p_{0} \rightarrow p_{0}$. Since $\models \alpha_{x} \rightarrow \tau(x), \models \mu\left(\alpha_{x}\right) \rightarrow \mu(\tau(x))$. Since $\mu\left(\alpha_{x}\right)=p_{0} \rightarrow p_{0}, \models \mu(\tau(x))$. Since $\models G_{x} \leftrightarrow(\bigwedge S \wedge x) \vee(\neg \wedge S \wedge \mu(\tau(x))), \models G_{x} \leftrightarrow(\bigwedge S \rightarrow x)$. Consequently, by

\footnotetext{
${ }^{5}$ Notice that for all $x \in \operatorname{var}(S)$, if $\alpha_{x}$ is a variable then $G_{x}$ is logically equivalent to the variant $(\bigwedge S \wedge x) \vee(\neg \bigwedge S \wedge \mu(\tau(x)))$ of the so-called Löwenheim formula [4, 13, 22].
} 
Proposition $4, G_{x}$ is implicative.

Case $\alpha_{x}$ is a parameter: Hence, $F_{x}=\alpha_{x}$. Thus, by Proposition $4, G_{x}$ is implicative. $\dashv$

Let $\epsilon$ be the substitution defined as follows:

- for all variables $x$, if $x \in \operatorname{var}(S)$ then $\epsilon(x)=G_{x}$,

- for all variables $x$, if $x \notin \operatorname{var}(S)$ then $\epsilon(x)=x$.

Note that for all variables $x$, if $x \in \operatorname{var}(S)$ then $\operatorname{var}(\epsilon(x)) \subseteq \operatorname{var}(S)$ and $\operatorname{par}(\epsilon(x)) \subseteq \operatorname{par}(S)$ else $\epsilon(x)=x$.

Lemma 3 For all variables $x, \models \tau(\epsilon(x)) \leftrightarrow \tau(x)$.

Proof: Let $x$ be a variable.

Case $x \in \operatorname{var}(S)$ : Hence, $\epsilon(x)=(\bigwedge S \wedge x) \vee(\neg \bigwedge S \wedge \mu(\tau(x))) \vee F_{x}$. Since $\tau$ is a unifier of $S, \models \tau(S)$. Since $\epsilon(x)=(\bigwedge S \wedge x) \vee(\neg \bigwedge S \wedge \mu(\tau(x))) \vee F_{x}$, $\models \tau(\epsilon(x)) \leftrightarrow \tau(x) \vee F_{x}$. Thus, by Lemma $1, \models \tau(\epsilon(x)) \leftrightarrow \tau(x)$.

Case $x \notin \operatorname{var}(S)$ : Hence, $\epsilon(x)=x$. Thus, $\models \tau(\epsilon(x)) \leftrightarrow \tau(x) . \dashv$

Lemma 4 For all $n \in \mathbb{N}$ and for all variables $x, \models \tau\left(\epsilon^{n}(x)\right) \leftrightarrow \tau(x)$.

Proof: By induction on $n$. $\dashv$

Lemma 5 For all $n \in \mathbb{N}, \epsilon^{n} \preceq_{\operatorname{var}(S)} \sigma$.

Proof: Let $n \in \mathbb{N}$. By Lemma $4, \epsilon^{n} \preceq_{\operatorname{var}(S)} \tau$. Since $\tau \preceq_{\operatorname{var}(S)} \sigma, \epsilon^{n} \preceq_{\operatorname{var}(S)} \sigma$. $\dashv$

Lemma 6 For all variables $x$, if $x \in \operatorname{var}(S)$ then $\models \bigwedge S \rightarrow\left(\epsilon(x) \leftrightarrow\left(x \vee F_{x}\right)\right)$ and $=\neg \bigwedge S \rightarrow(\epsilon(x) \leftrightarrow \mu(\tau(x)))$.

Proof: Let $x$ be a variable such that $x \in \operatorname{var}(S)$. Hence, $\epsilon(x)=(\bigwedge S \wedge x) \vee$ $(\neg \bigwedge S \wedge \mu(\tau(x))) \vee F_{x}$. Thus, $\models \bigwedge S \rightarrow\left(\epsilon(x) \leftrightarrow\left(x \vee F_{x}\right)\right)$ and $\models \neg \bigwedge S \rightarrow$ $\left(\epsilon(x) \leftrightarrow\left(\mu(\tau(x)) \vee F_{x}\right)\right)$. Consequently, by Lemma $1, \models \neg \bigwedge S \rightarrow(\epsilon(x) \leftrightarrow$ $\mu(\tau(x))) . \dashv$

Lemma 7 For all formulas $\varphi$, if $\operatorname{var}(\varphi) \subseteq \operatorname{var}(S)$ then $\models \neg \bigwedge S \rightarrow(\epsilon(\varphi) \leftrightarrow$ $\mu(\tau(\varphi)))$.

Proof: By induction on $\varphi$. $\dashv$

Lemma 8 For all variables $x$, if $x \in \operatorname{var}(S)$ then $\models(x \wedge \mu(\tau(x))) \rightarrow \epsilon(x)$ and $\models \epsilon(x) \rightarrow(x \vee \mu(\tau(x)))$. 
Proof: Let $x$ be a variable such that $x \in \operatorname{var}(S)$. By Lemma $6, \models \wedge S \rightarrow$ $\left(\epsilon(x) \leftrightarrow\left(x \vee F_{x}\right)\right)$ and $\models \neg \bigwedge S \rightarrow(\epsilon(x) \leftrightarrow \mu(\tau(x)))$. Hence, $\models \wedge S \rightarrow((x \wedge$ $\mu(\tau(x))) \rightarrow \epsilon(x))$ and $=\neg \wedge S \rightarrow((x \wedge \mu(\tau(x))) \rightarrow \epsilon(x))$. Thus, $\models(x \wedge$ $\mu(\tau(x))) \rightarrow \epsilon(x)$. Since $=\bigwedge S \rightarrow\left(\epsilon(x) \leftrightarrow\left(x \vee F_{x}\right)\right)$ and $\models \neg \bigwedge S \rightarrow(\epsilon(x) \leftrightarrow$ $\mu(\tau(x))), \models \bigwedge S \rightarrow\left(\epsilon(x) \rightarrow\left(x \vee F_{x} \vee \mu(\tau(x))\right)\right)$ and $\models \neg \bigwedge S \rightarrow(\epsilon(x) \rightarrow$ $\left.\left(x \vee F_{x} \vee \mu(\tau(x))\right)\right)$. Consequently, $=\epsilon(x) \rightarrow\left(x \vee F_{x} \vee \mu(\tau(x))\right)$. Hence, by Lemma $1, \models \epsilon(x) \rightarrow(x \vee \mu(\tau(x))) . \dashv$

Lemma $9 \models \neg \bigwedge S \rightarrow \epsilon(\bigwedge S)$.

Proof: By Lemma $7, \models \neg \bigwedge S \rightarrow(\epsilon(\bigwedge S) \leftrightarrow \mu(\tau(\bigwedge S)))$. Since $\tau$ is a unifier of $S, \models \tau(S)$. Hence, $\models \mu(\tau(S))$. Since $\models \neg \bigwedge S \rightarrow(\epsilon(\bigwedge S) \leftrightarrow \mu(\tau(\bigwedge S)))$, $\models \neg \bigwedge S \rightarrow \epsilon(\bigwedge S) . \dashv$

Lemma 10 For all $n \in \mathbb{N}$ and for all variables $x$, if $n \geq\|\operatorname{var}(S)\|$ and $x \in$ $\operatorname{var}(S)$ then $\models \epsilon\left(\epsilon^{n}(x)\right) \leftrightarrow \epsilon^{n}(x)$.

Proof: Let $n \in \mathbb{N}$ and $x$ be a variable such that $n \geq\|\operatorname{var}(S)\|$ and $x \in \operatorname{var}(S)$. By Lemma $8, \models(x \wedge \mu(\tau(x))) \rightarrow \epsilon(x)$ and $\models \epsilon(x) \rightarrow(x \vee \mu(\tau(x)))$. Hence, $\models \mu(\tau(x)) \rightarrow(x \rightarrow \epsilon(x))$ and $\models \mu(\tau(x)) \vee(\epsilon(x) \rightarrow x)$. Since $n \geq\|\operatorname{var}(S)\|$, by Propositions 6 and $7, \models \mu(\tau(x)) \rightarrow\left(\epsilon\left(\epsilon^{n}(x)\right) \leftrightarrow \epsilon^{n}(x)\right)$ and $\models \mu(\tau(x)) \vee$ $\left(\epsilon\left(\epsilon^{n}(x)\right) \leftrightarrow \epsilon^{n}(x)\right)$. Thus, $\models \epsilon\left(\epsilon^{n}(x)\right) \leftrightarrow \epsilon^{n}(x) . \dashv$

Lemma 11 For all $n \in \mathbb{N}$, if $n \geq\|\operatorname{var}(S)\|$ then $\epsilon^{n}$ is a unifier of $S$.

Proof: Let $n \in \mathbb{N}$ be such that $n \geq\|\operatorname{var}(S)\|$. By Lemma $9, \models \neg \bigwedge S \rightarrow \epsilon(\bigwedge S)$. Hence, $\models \bigwedge S \vee \epsilon(\bigwedge S)$. Thus, $\models \epsilon^{n}(\bigwedge S) \vee \epsilon^{n}(\epsilon(\bigwedge S))$. Consequently, by Proposition $5, \models \epsilon^{n}(\bigwedge S) \vee \epsilon\left(\epsilon^{n}(\bigwedge S)\right)$. Since $n \geq\|\operatorname{var}(S)\|$, by Lemma 10 , $\models \epsilon\left(\epsilon^{n}(\bigwedge S)\right) \leftrightarrow \epsilon^{n}(\bigwedge S)$. Since $\models \epsilon^{n}(\bigwedge S) \vee \epsilon\left(\epsilon^{n}(\bigwedge S)\right), \models \epsilon^{n}(S)$

Proposition 9 Let $S \subseteq$ FOR be finite. If $S$ is unifiable then either $S$ is unitary, or $S$ is finitary.

Proof: Suppose $S$ is unifiable.

Case $\operatorname{par}(S)=\emptyset$ : Let $\epsilon$ be the substitution defined by

- for all variables $x$, if $x \in \operatorname{var}(S)$ then $\epsilon(x)=\bigwedge S \rightarrow x$,

- for all variables $x$, if $x \notin \operatorname{var}(S)$ then $\epsilon(x)=x$.

The reader may easily verify that $\epsilon$ constitutes by itself a minimal complete set of unifiers of $S[10,24]$. Hence, $S$ is unitary.

Case $\operatorname{par}(S) \neq \emptyset$ : Let $p_{0}$ be a parameter in $S$. Let $\Sigma$ be the set of all unifiers of $S$. Note that $\Sigma$ is complete. Let $\Sigma^{\prime}$ be the set of unifiers of $S$ obtained from $\Sigma$ by keeping only the unifiers $\sigma$ of $S$ such that $\operatorname{var}(\sigma(S)) \subseteq \operatorname{var}(S)$ and $\operatorname{par}(\sigma(S)) \subseteq \operatorname{par}(S)$. Since $\Sigma$ is complete, by Lemmas 5 and $11, \Sigma^{\prime}$ is complete. 
Let $\Sigma^{\prime \prime}$ be the set of unifiers of $S$ obtained from $\Sigma^{\prime}$ by keeping only one representative of each equivalence class modulo $\simeq_{\operatorname{var}(S)}$. Since $\Sigma^{\prime}$ is complete, $\Sigma^{\prime \prime}$ is complete. Moreover, $\Sigma^{\prime \prime}$ is finite. Thus, either $S$ is unitary, or $S$ is finitary. $\dashv$

Proposition 10 Let $n \geq 1$ and $S=\left\{x \rightarrow p_{1} \vee \ldots \vee p_{n}\right\}$

1. $S$ is unifiable,

2. if for all $i, j \in\{1, \ldots, n\}, p_{i}=p_{j}$ then $S$ is unitary,

3. if there exists $i, j \in\{1, \ldots, n\}$ such that $p_{i} \neq p_{j}$ then $S$ is finitary.

\section{Proof:}

(1). For all $i \in\{1, \ldots, n\}$, let $\sigma_{i}$ be the substitution defined as follows:

- $\sigma_{i}(x)=p_{i}$,

- for all variables $y$, if $y \neq x$ then $\sigma_{i}(y)=y$.

Obviously, for all $i \in\{1, \ldots, n\}, \sigma_{i}$ is a unifier of $S$. Hence, $S$ is unifiable.

(2). Suppose for all $i, j \in\{1, \ldots, n\}, p_{i}=p_{j}$. Let $p$ be the parameter such that for all $i \in\{1, \ldots, n\}, p=p_{i}$ and $\sigma$ be the substitution defined as follows:

- $\sigma(x)=p$,

- for all variables $y$, if $y \neq x$ then $\sigma(y)=y$.

Obviously, $\sigma$ is a unifier of $S$. Moreover, for all unifiers $v$ of $S, \models v(x) \rightarrow p$ and by Proposition 2, $\models p \rightarrow v(x)$. Thus, for all unifiers $v$ of $S, \models v(x) \leftrightarrow \sigma(x)$. Consequently, for all unifiers $v$ of $S, v \simeq_{\operatorname{var}(S)} \sigma$. Hence, $S$ is unitary.

(3). Suppose there exists $i, j \in\{1, \ldots, n\}$ such that $p_{i} \neq p_{j}$. For the sake of the contradiction, suppose $S$ is not finitary. Thus, by Proposition $9, S$ is unitary. Let $v$ be a unifier of $S$ such that for all $k \in\{1, \ldots, n\}, v \preceq_{\operatorname{var}(S)} \sigma_{k}$. Thus, $\models v(x) \rightarrow p_{1} \vee \ldots \vee p_{n}$ and for all $k \in\{1, \ldots, n\}$, there exists a substitution $\theta_{k}$ such that $=\theta_{k}(v(x)) \leftrightarrow p_{k}$. Consequently, by Proposition 2 , let $l \in\{1, \ldots, n\}$ be such that $\models p_{l} \rightarrow v(x)$. Hence, $\models p_{l} \rightarrow \theta_{i}(v(x))$ and $\models p_{l} \rightarrow \theta_{j}(v(x))$. Since for all $k \in\{1, \ldots, n\}, \models \theta_{k}(v(x)) \leftrightarrow p_{k}, \models p_{l} \rightarrow p_{i}$ and $\models p_{l} \rightarrow p_{j}$. Thus, $p_{l}=p_{i}$ and $p_{l}=p_{j}$. Consequently, $p_{i}=p_{j}$ : a contradiction. Hence, $S$ is finitary.

From Propositions 9 and 10, it follows that

Theorem 1 The implication fragment of Classical Propositional Logic is finitary for unification with parameters, i.e.

- for all finite $S \subseteq \mathbf{F O R}$, if $S$ is unifiable then either $S$ is unitary, or $S$ is finitary,

- there exists a finite $S \subseteq$ FOR such that $S$ is unifiable and $S$ is finitary, 


\section{Conclusion}

In this paper, we have seen that, in contrast to the full language of CPL which is unitary both for elementary unification and for unification with parameters $[4,13,22]$, the implication fragment of CPL is unitary for elementary unification and finitary for unification with parameters. Below, we propose numerous conjectures and varied problems, with the hope of setting goals for future research in unification theory.

It is known that when CPL is based on a definitionally complete set of connectives, elementary unification is NP-complete and unification with parameters is $\Pi_{2}^{\mathbf{P}}$-complete [3]. From Section 4, it follows that elementary unification in the implication fragment of CPL is trivial and unification with parameters in the implication fragment of $\mathbf{C P L}$ is decidable. But what is the complexity of unification with parameters in the implication fragment of CPL?

As noted in the introduction, the rule $\frac{x \rightarrow p}{p \rightarrow x}$ is admissible in the implication fragment of CPL. Nevertheless, it is not derivable. Hence, the question arises: for the implication fragment of $\mathbf{C P L}$, does the set of all admissible rules admit a finite basis, i.e. a finite set of rules that added to the axiomatization of the implication fragment of CPL mentioned in the introduction exactly produces the set of all admissible rules ${ }^{6}$ ?

For a modal language based on the connectives $\rightarrow, \neg$ and $\square$, it is known, for example, that $\mathbf{S} 5$ is unitary [12], $\mathbf{S} 4$ is finitary [17, 20] and $\mathbf{K}$ is nullary [21]. This immediately leads us to ask what is the unification type of $\mathbf{S} 5, \mathbf{S} 4$ and $\mathbf{K}$ when $\rightarrow$ and $\square$ are the sole connectives? This question is of course related to the question of the unification type of some of the modal algebras discussed by Celani and Montangie [7, 8]. It is not a trivial question. For instance, the formula $x \rightarrow \square x$ used by Jerábek [21] to show that $\mathbf{K}$ is nullary becomes unitary when $\rightarrow$ and $\square$ are the sole connectives ${ }^{7}$.

Remind that in a modal language based on the connectives $\rightarrow$, $\neg$ and $\square$, the connective of strict implication is defined as follows: $(\varphi \Rightarrow \psi)::=\square(\varphi \rightarrow \psi)[18$, Chapter 2]. Hence, there is also the question of the unification type of the frag-

\footnotetext{
${ }^{6}$ See [19] for a definition of what a basis of admissible rules is within the context of Intuitionistic Propositional Logic.

${ }^{7}$ In order to understand why, it suffices to notice that

- the substitution $\sigma$ defined as follows:

$-\sigma(x)=x \rightarrow x$,

- for all variables $y$, if $x \neq y$ then $\sigma(y)=y$,

is a $\mathbf{K}$-unifier of $x \rightarrow \square x$,

- for all modal formulas $\varphi$ with $\rightarrow$ and $\square$ as its sole connectives, if $\varphi \rightarrow \square \varphi$ is in $\mathbf{K}$ then $\varphi$ is in $\mathbf{K}$.
} 
ments of the above-mentioned modal logics when $\Rightarrow$ is the sole connective. For those who interest in such fragments which have been extensively studied over the years, we recommend the reading of [14].

\section{Funding}

The preparation of this paper has been supported by French Minister of Europe and Foreign Affairs, French Minister of Higher Education, Research and Innovation and Iranian Minister of Research, Science and Technology (Project 40903VF).

\section{Acknowledgement}

Special acknowledgement is heartily granted to Joseph Boudou (Paul Sabatier University, Toulouse, France), Çiğdem Gencer (Istanbul Aydın University, Istanbul, Turkey), Maryam Rostamigiv (Paul Sabatier University, Toulouse, France) and Tinko Tinchev (Sofia University St. Kliment Ohridski, Sofia, Bulgaria) for many stimulating discussions about the unification problem. We also make a point of strongly thanking the referees for their feedback: their useful suggestions have been essential for improving the correctness and the readability of a preliminary version of this paper.

\section{References}

[1] Аввотт, J., 'Implicational algebras', Bulletin Mathématique de la Société des Sciences Mathématiques de la République Socialiste de Roumanie 11:3-23, 1967.

[2] Anantharaman, S., P. Narendran, and M. Rusinowitch, 'Unification modulo ACUI plus distributivity axioms', Journal of Automated Reasoning $33: 1-28,2004$.

[3] BaAder, F., 'On the complexity of Boolean unification', Information Processing Letters 67:215-220, 1998.

[4] BAAder, F., and S. Ghilardi, 'Unification in modal and description logics', Logic Journal of the IGPL 19 (2011) 705-730.

[5] BaAder, F., and W. Snyder, 'Unification theory', In: Handbook of Automated Reasoning, Elsevier (2001) 439-526.

[6] Blackburn, P., M. De Rijke, and Y. Venema, Modal Logic, Cambridge University Press (2001).

[7] Celani, S., and D. Montangie, 'Hilbert algebras with a necessity modal operator', Reports on Mathematical Logic 49:47-77, 2014. 
[8] Celani, S., and D. Montangie, 'Hilbert algebras with a modal operator $\diamond^{\prime}$, Studia Logica 103:639-662, 2015.

[9] Chagrov, A., and M. Zakharyaschev, Modal Logic, Oxford University Press (1997).

[10] Cintula, P., and G. Metcalfe, 'Admissible rules in the implicationnegation fragment of intuitionistic logic', Annals of Pure and Applied Logic 162 (2010) 162-171.

[11] Dunn, M., and G. Hardegree, Algebraic Methods in Philosophical Logic, Oxford University Press (2001).

[12] DzIK, W., 'Unitary unification of $\mathbf{S} 5$ modal logics and its extensions', Bulletin of the Section of Logic 32 (2003) 19-26.

[13] DzIK, W., Unification Types in Logic, Wydawnicto Uniwersytetu Slaskiego (2007).

[14] Ernst, Z., B. Fitelson, K. Harris, and L. Wos, 'Shortest axiomatizations of implicational $\mathbf{S} 4$ and $\mathbf{S} 5$ ', Notre Dame Journal of Formal Logic $\mathbf{4 3}$ (2002) 169-179.

[15] Ghilardi, S., 'Unification through projectivity', Journal of Logic and Computation 7 (1997) 733-752.

[16] Ghilardi, S., 'Unification in intuitionistic logic', Journal of Symbolic Logic 64 (1999) 859-880.

[17] Ghilardi, S., 'Best solving modal equations', Annals of Pure and Applied Logic 102 (2000) 183-198.

[18] Hughes, G., and M. Cresswell, An Introduction to Modal Logic, Methuen (1968).

[19] Iemhoff, R., 'On the admissible rules of intuitionistic propositional logic', Journal of Symbolic Computation 66:281-294, 2001.

[20] IEmhoff, R., 'A syntactic approach to unification in transitive reflexive modal logics', Notre Dame Journal of Formal Logic 57 (2016) 233-247.

[21] JER̆ÁBEK, E., 'Blending margins: the modal logic $\mathbf{K}$ has nullary unification type', Journal of Logic and Computation 25 (2015) 1231-1240.

[22] Martin, U., and T. Nipkow, 'Boolean unification - the story so far', Journal of Symbolic Computation 7 (1989) 275-293.

[23] Papadimitriou, C., Computational Complexity. Addison-Wesley (1995).

[24] Prucnal, T., 'On the structural completeness of some pure implicational propositional calculi', Studia Logica 30 (1972) 45-50. 
[25] Rybakov, V., Admissibility of Logical Inference Rules, Elsevier (1997).

[26] Wolter, F., and M. Zakharyaschev, 'Undecidability of the unification and admissibility problems for modal and description logics', ACM Transactions on Computational Logic 9:25:1-25:20, 2008. 